\title{
Acute disseminated encephalomyelitis in dengue viral infection
}

\begin{abstract}
Dengue is the most common arboviral disease affecting many countries worldwide. An RNA virus from the flaviviridae family, dengue has four antigenically distinct serotypes (DEN-1DEN-4). Neurological involvement in dengue can be classified into dengue encephalopathy immune-mediated syndromes, encephalitis, neuromuscular or dengue muscle dysfunction and neuro-ophthalmic involvement. Acute disseminated encephalomyelitis (ADEM) is an immune mediated acute demyelinating disorder of the central nervous system following recent infection or vaccination. This monophasic illness is characterised by multifocal white matter involvement. Many dengue studies and case reports have linked ADEM with dengue virus infection but the association is still not clear. Therefore, this article is to review and discuss concerning ADEM in dengue as an immune-medicated neurological complication; and the management strategy required based on recent literature.
\end{abstract}

Keyword: Acute disseminated; Demyelinating diseases; Dengue virus; Encephalomyelitis; Nervous system disorders; Severe dengue 